\section{$\underset{\substack{\text { hommes } \\ \text { \& migrations }}}{ }$}

\section{Hommes \& migrations}

Revue française de référence sur les dynamiques

migratoires

$1309 \mid 2015$

Le $3 e$ âge des migrants

\title{
Le vieillissement des immigrés : état des savoirs
}

\section{Mohamed Madoui}

\section{(2) OpenEdition}

Journals

\section{Édition électronique}

URL : http://journals.openedition.org/hommesmigrations/3061

DOI : 10.4000/hommesmigrations.3061

ISSN : 2262-3353

\section{Éditeur}

Musée national de l'histoire de l'immigration

\section{Édition imprimée}

Date de publication : 1 janvier 2015

Pagination : 11-17

ISBN : 978-2-919040-30-8

ISSN : $1142-852 X$

\section{Référence électronique}

Mohamed Madoui, «Le vieillissement des immigrés : état des savoirs », Hommes \& migrations [En ligne], 1309 | 2015, mis en ligne le 01 janvier 2017, consulté le 20 avril 2019. URL : http:// journals.openedition.org/hommesmigrations/3061; DOI : 10.4000/hommesmigrations.3061 


\title{
LE VIEILLISSEMENT DES IMMIGRÉS ÉTAT DES SAVOIRS
}

par MOHAMED MADOUI, professeur des universités au Cnam.

\author{
Depuis une quarantaine d'années, la question du vieillissement \\ des migrants sur le territoire français mobilise la recherche \\ universitaire. Deux ensembles de questions se dégagent. \\ Les conditions de vie des immigrés nord-africains, notamment \\ en foyer, ont tout d'abord occupé les chercheurs, avec leur lot \\ de discriminations. Les recherches prennent aujourd'hui \\ davantage en compte les liens familiaux pour comprendre \\ les raisons du non-retour au pays d'origine.
}

Si la question de l'intégration des immigrés et de leurs descendants a été largement abordée, celle des retraités et des migrants vieillissants ${ }^{1}$ a longtemps été négligée tant par les pouvoirs publics à cause sans doute du désintérêt pour le vieux migrant devenu "improductif", que par le monde académique plus préoccupé par la question de l'intégration à la société française de descendants d'immigrés. À l'exception de quelques associations très actives en matière de défense et de soutien des travailleurs migrants comme le Groupe d'information et de soutien des immigrés (Gisti) ou la Cimade (Comité inter-mouvements auprès des évacués - service œcuménique d’entraide), le secteur associatif n'a pas fait de la problématique des vieux migrants une cause prioritaire et mobilisatrice, malgré de fortes inégalités d'accès aux droits sociaux et à la protection sociale des migrants vieillissants ${ }^{2}$.

En dehors des travaux conduits par des démographes et des statisticiens à la fin des années 1970 portant essentiellement sur le poids des travailleurs migrants sur le vieillissement de la population française $^{3}$, les premières recherches sur les migrants 
âgés remontent aux années 1980 et portaient notamment sur la santé et l'équilibre social chez les immigrés ${ }^{4}$. Le colloque Psychologie médicale et migrants qui s'est tenu à Marseille en 1980, suivi six ans plus tard, en 1986, d'un autre colloque organisé à l'initiative du Centre interdisciplinaire de gérontologie, "La vieillesse des étrangers en France", constituait le point de départ du débat sur le vieillissement de la population immigrée et mettent en évidence les problèmes posés par la retraite du travailleur migrant. La revue Gérontologie consacrait la même année un dossier à cette thématique du vieillissement des migrants, dans lequel on trouve un article intéressant d'Abdelmalek Sayad intitulé : "La vacance comme pathologie de la contradiction d'immigré : le cas de la retraite et de la préretraite ${ }^{5}$."

\section{La sociologie au service de la prise de conscience des pouvoirs publics}

Il faudra attendre le début des années 1990 pour que les pouvoirs publics prennent conscience de la problématique du vieillissement migrant. Le Fonds d'action sociale (FAS) qui dépend du ministère des Affaires sociales commande, en 1992, un rapport de recherche sur le vieillissement des immigrés en région parisienne. Cette étude sera conduite sous la direction de l'historien Gérard Noiriel et donnera lieu à la publication d'un ouvrage collectif intitulé Vieillir et mourir en exil. Immigration maghrébine et vieillissement ${ }^{6}$, dans lequel ont été traitées les multiples ruptures vécues par le migrant et les souffrances physiques et psychologiques dans lesquelles sont plongés une grande partie des migrants âgés. Dans la même foulée, une revue grenobloise Écarts d'identité consacre en 1993 un numéro entier ${ }^{7}$ à la question du vieillissement des immigrés, en abordant de façon empirique et pragmatique les difficultés auxquelles font face les retraités, notamment en matière de logement, de l'accès aux soins et à d'autres droits sociaux ${ }^{8}$.

Sur le plan de l'égalité des droits entre Français et immigrés dans le domaine de la protection sociale, un collectif réunissant un ensemble d'associations ${ }^{9}$ met sur le devant de la scène les migrants âgés ou handicapés et publie en 1994 une brochure "pour une égalité de traitement : les engagements internationaux de la France pour les handicapés et les retraités étrangers". Le Gisti va reprendre ce document pour en faire un numéro spécial de sa revue Plein droit qui porte un titre explicite, "Une vieillesse illégitime", en listant un ensemble de problèmes spécifiques liés aux conditions de logement, à la protection sociale et à l'accès aux droits ${ }^{10}$.

La question des migrants vieillissants s'est construite en réalité grâce à l'action efficace de quelques associations et de chercheurs militants venant de champs disciplinaires variés (gérontologie, sociologie, démographie, histoire, droit, intervention sociale, etc.). Des revues comme Écarts d'identité et Plein droit, suivies par Hommes \& Migrations et Migrations Société, ont permis de rendre visible cette question du vieillissement migrant en alimentant la presse spécialisée et les débats de société. Cet intérêt pour les migrants vieillissants gagne aussi les pouvoirs publics, qui organisent en mai 1999, à l'initiative du FAS, un colloque national à l'université d'Aix-en-Provence sur le thème de "Vieillesse et immigration", auquel la revue la revue Migrations Société consacra un remarquable dossier ${ }^{11}$.

\footnotetext{
4. Abdelmalek Sayad, "Santé et équilibre social chez les immigrés", in Psychologie médicale, n 11, tome 13, 1981, pp. 1747-1775. 5. Abdelmalek Sayad, "La vacance comme pathologie de la contradiction d'immigré. Le cas de la retraite et de la préretraite", in Gérontologie, n 60, 1986, pp. 37-55. 6. Gérard Noiriel (dir), "Le vieillissement des immigrés en Région parisienne. Rapport final", Fas, Paris, 1993. 7. Écarts d'identité, n64, "Vieillir dans l'immigration”, 1993. 8. Gilles Dubus, Françoise Braud, "Les migrants âgés dans les publications scientifiques francophones", in Revue européenne des migrations internationales, vol. 17, $\mathrm{n}^{\circ}$ 1, 2001, pp. 189-197. 9. Le Collectif des accidentés du travail, handicapés et retraités pour l'égalité des droits (Catred), la Fédération nationale des accidentés du travail et des handicapés (FNATH), le Groupe d'information et de soutien des travailleurs immigrés (Gisti), le Groupe de recherche et d'action sur le vieillissement des étrangers en France (GRAVE) et l'Office dauphinois des travailleurs immigrés (ODTI). 10. Gilles Dubus, Françoise Braud, op. cit. 11. Migrations Société, vol.12, n 68, 2000.
} 
Foyer Adoma “Les Grésillons" à Gennevilliers. Ici, plusieurs chambres sont occupées par des chibanis. Parmi eux, certains sont là depuis plus de trente ans, $1^{\text {er }}$ avril 2015 ( CAmille Millerand

\section{Immigrés algériens : vieillir sous surveillance}

Les sociologues de l'immigration vont peu à peu investir la problématique de la vieillesse des publics migrants grâce à des enquêtes de terrain, à des témoignages et à des monographies. Aux côtés des travaux d'Abdelmalek Sayad ${ }^{12}$ et de Gérard Noiriel ${ }^{13}$ qui ont montré que le temps de la retraite est souvent un moment de profonde remise en question de l'identité des vieux migrants, dont seul le travail justifiait jusqu'ici leur présence en France, d’autres sociologues, à l'instar de Marc Bernardot ${ }^{14}$ et de Rémi Gallou ${ }^{15}$, se sont intéressés aux conditions de logement et de vieillissement des migrants résidant dans des foyers Sonacotra (rebaptisés foyers Adoma en 2007). Ces foyers ont été conçus à l'origine pour deux raisons, toutes deux discriminatoires : produire du sous-logement et faire vivre les immigrés à l'écart de la société ${ }^{16}$. Les pouvoirs publics considéraient ce type de logement comme provisoire mais ont continué paradoxalement à en construire sur le même modèle jusqu'au début des années 1980. Ainsi, de 1966 à 1976, la Société nationale

12. Abdelmalek Sayad, "La vacance comme pathologie de la contradiction d'immigré. Le cas de la retraite et de la préretraite", op. cit.; Abdelmalek Sayad, L'Immigration ou les paradoxes de l'altérité, Bruxelles, De Boeck, 1991. 13. Gérard Noiriel (dir.), op. cit.. 14. Marc Bernardot, "Chronique d'une institution : la Sonacotra (1956-1976)", in Sociétés contemporaines, n 33-34, 1999, pp. 3958 ; Marc Bernardot, "Le vieux, le fou et l'autre. Qui habite encore dans les foyers ?", in Revue européenne des migrations internationales, vol. 1, $n^{\circ}$ 17, 2001, pp. 151-164 ; Marc Bernardot, "Déplacer et loger les indésirables, sociologie du logement contraint", working paper, 2005, disponible sur http://www.reseau-terra.eu/article.php3?id_article=337; Marc Bernardot, "Voyage dans la chambre noire. Les foyers de travailleurs migrants à Paris", in Hommes \& Migrations, n 1264, 2006, pp. 57-67. 15. Rémi Gallou, "Les immigrés isolés : la spécificité des résidents en foyer", in Retraite et société, n 44, 2005, pp. 108-147. 16. Voir le rapport de la Mission d'information sur les immigrés âgés, Assemblée nationale, 2 juillet 2013. 
de construction de logements pour les travailleurs algériens (Sonacotral) a construit plus de 200 foyers de grande taille sur le modèle de la haute tour de 13 étages ne comportant qu'une seule entrée.

Dans les années 1960, les immigrés algériens sont les seuls à faire l'objet d'une offre de logement spécifique et d'un encadrement par les pouvoirs publics, qui estiment, comme le souligne à juste titre Choukri Hmed, que "ces Nord-Africains d'origine rurale, 'inadaptés' à la ville, rétifs à l'individualisme comme à la raison, doivent être pris en charge au sein d'institutions qui débordent le strict cadre du travail afin qu'ils puissent s'acculturer à la civilisation occidentale et abandonner le caractère primitif et instable de leur 'mentalitén ${ }^{17}$ ". Il y a véritablement une logique ségrégative des politiques de logement conduites à l'égard des immigrés algériens, qui seront étendues par la suite à tous les travailleurs migrants. À l'opposé du modèle du "repos
L'isolement et la précarité sont

exacerbés au moment

de la retraite par des conditions

précaires de logement,

par des revenus faibles ne

permettant pas de se loger

à un prix décent dans le parc

social ou privé, et par

des problèmes de santé liés à la pénibilité de leurs activités mérité", "ces vieux, comme le souligne Marc Bernardot, ne savent vraiment pas comment ils sont entrés dans ce nouvel 'âge de la vie', subissant la remise en cause de leurs droits par leurs employeurs, les organismes de retraite et même par les autorités publiques ${ }^{18}$. Les travaux de Marc Bernardot ${ }^{19}$ mettent également en évidence la quasi-absence de relais associatifs et de réseaux sociaux ou syndicaux, laissant cette population dans un isolement total. Ils ne sont même plus membres d'anciennes structures d'encadrement comme l'Amicale des Algériens en Europe ou Les Marocains résidents de l'étranger (MRE).

Le foyer devient ainsi synonyme d'isolement social, un espace aussi hostile que le monde extérieur. Ces foyers étaient pensés au départ comme un moyen de se débarrasser des bidonvilles qui, dans le contexte de la guerre d’Algérie, font craindre aux pouvoirs publics l'agitation politique et la montée des mouvements insurrectionnels en métropole en faveur du Front de libération nationale $(\mathrm{FLN})^{20}$. De lieux d'habitation provisoires, ils vont devenir avec le temps des logements durables où "les hommes ont vieilli dans les murs" 21 " et où l'on a confiné des migrants n'ayant pas réussi à rentrer définitivement au pays. Parmi les migrants habitant en foyer en 1999, six sur dix y habitaient déjà en 1974. Comme le remarque Rémi Gallou, les foyers sont devenus des "non-lieux", autrement dit des espaces construits dans l'urgence, qui ne sont ni le lieu d'une immigration provisoire, ni des lieux de vie pour les retraités migrants durablement installés ${ }^{22}$.

L'isolement et la précarité sont exacerbés au moment de la retraite par des conditions précaires de logement, par des revenus faibles ne permettant pas de se loger à un prix décent dans le parc social ou privé, et par des problèmes de santé liés à la pénibilité de leurs activités. À cela s'ajoutent les pratiques d'encadrement de ces foyers dirigés en majorité par des anciens militaires ayant fait la guerre d'Algérie et à qui on a donné la mission de "tenir leur hommes ${ }^{23}$ ". Pour maintenir l'ordre dans ces foyers, leurs directeurs adoptent des méthodes de surveillance et de coercition dignes de l'expérience de commandement des hommes en période de guerre. Des méthodes justifiées au nom de la sécurité et de la tranquillité des résidents, "considérés comme incapables - par leurs déterminismes culturels - de faire régner l'ordre ${ }^{24 "}$. Certaines études, comme le montre le tableau ci-dessous, ont mis en évidence le caractère "ethnicisé" de la gestion des foyers et de leurs résidents, qui sont le plus souvent regroupés par étage, en fonction de leur origine ethnique et de leur provenance.

17. Choukri Hmed, “'́encadrement des étrangers 'isolés' par le logement social (1950-1980)", in Genèses, $n^{\circ} 72,2008$, pp. 63-81. 18. Marc Bernardot, “Chronique d'une institution : la Sonacotra (1956-1976)”, op. cit. 19. Ibid. ; Marc Bernardot, “Le vieux, le fou et l'autre. Qui habite encore dans les foyers?", op. cit 20. Choukri Hmed, "Tenir ses hommes. La gestion des étrangers isolé dans les foyers Sonacotra après la guerre d'Algérie", in Politix, $n^{\circ} 76,2006$, pp. 11-30. 21. Rémi Gallou, “Le vieillissement des immigrés en France : le cas paroxystique des résidents des foyers”, in Politix, n 72, 2005, pp. 55-77. 22. Ibid., p.72. 23. Choukri Hmed, "Tenir ses hommes. La gestion des étrangers isolés dans les foyers Sonacotra après la guerre d'Algérie", op. cit. Selon les statistiques de la Sonacotra en 1972, sur 151 directeurs de foyer, 143 étaient passés par les armées coloniales. 
/// La gestion "ethnicisée" des résidents d'un foyer en Seine-Saint-Denis en 1992

\begin{tabular}{|c|c|c|c|c|}
\hline Étage & Chambres & Douches & Escaliers & Sanitaires \\
\hline $4^{\mathrm{e}}$ étage & Soninkés & & & \\
\hline $3^{e}$ étage & Bambaras & & & \\
\hline $2^{2}$ étage & Wolofs & & & \\
\hline $1^{\text {er étage }}$ & Maghrébins & & & \\
\hline Rez-de-chaussée & Mosquée-TV & & Hall & \\
\hline
\end{tabular}

Source : Xavier Vandromme, Vieillir immigré et célibataire en foyer. Le cas de la résidence sociale du Bourget en Seine-Saint-Denis (1990-1992), Paris, Ciemi, L'Harmattan, 1996, p. 39.

\section{Le non-retour au pays d'origine, un facteur paradoxal d'intégration}

D'autres travaux de recherche ont abordé les migrants vieillissants au temps de la retraite. Allant à contre-courant del'idée d'une "vieillesse illégitime" et des discours catastrophistes à l'égard des vieux migrants, Claudine Attias-Donfut ${ }^{25}$ considère, au contraire, la retraite comme un facteur d'intégration : "Loin d'être ressenti comme illégitime, rester en France au temps de la retraite est au contraire un facteur d'intégration. Le rapport à la vie de retraité est conditionné en priorité par l'état de santé et le niveau de revenu, ce qui est le cas de tout immigré ou non ${ }^{26 . "}$ En s'appuyant sur une enquête réalisée en collaboration avec la Caisse nationale d'assurance vieillesse (Cnav) et l'Institut national de la statistique et des études économiques (Insee) portant sur le vieillissement et le passage à la retraite des immigrés en France ${ }^{27}$, Claudine Attias-Donfut constate que globalement les immigrés trouvent plus d'avantages que d'inconvénients à la retraite: "Une majorité (59\%) y trouve à la fois des avantages et des inconvénients, une forte minorité n'y voit que des avantages $(27 \%)$ et pour une petite minorité (13,4\%), la retraite ne présente que des inconvénients." Plus de la moitié des immigrés considèrent que le passage à la retraite n'a pas entraîné une diminution de leur niveau de vie, ce qui va à l'encontre des propos que nous avons recueillis auprès de vieux migrants rencontrés au café social de Belleville, qui se plaignent plutôt de leurs difficultés à boucler les fins de mois avec leurs maigres pensions. D'après la même enquête, les retraités migrants sont également peu nombreux à envisager de rentrer définitivement dans leur pays d'origine $(1,7 \%)$ et une très grande majorité d'entre eux (70 \%) ne ressent aucune nostalgie du pays d'origine. Sans entrer dans les débats philosophiques sur la notion de nostalgie, celle-ci est évidemment à relativiser, car nombreux sont les retraités qui circulent et effectuent des séjours réguliers dans leur pays d’origine. Malgré les conditions vétustes de logement, la grande majorité des immigrés interrogés avant lâge de la retraite souhaitent rester vivre en France (60 \%), tandis que $23 \%$ d'entre eux préfèrent partager leur temps et circuler entre la France et leur pays d'origine. Il n'y a que $6 \%$ des personnes interrogées qui souhaitent retourner définitivement dans leur pays d'origine. Ces chiffres sur le non-retour au pays d'origine étonnent également et ne vont pas dans le sens des estimations du Haut Conseil à l'intégration qui souligne dans son rapport de 2002 que plus 
de 90000 personnes ont quitté la France dans les dix dernières années pour s'établir et toucher leur retraite en Algérie, soit un flux de retour d'environ 9100 personnes par an, sans doute le plus important comparé aux Marocains (1 400 départs/an), les Espagnols (800 départs/an), les Portugais (3 800 départs/an) et les Italiens (1 300 départs/an). Aucun indicateur ne permet de dire de façon tranchée que les personnes qui décident de rentrer définitivement dans leur pays d'origine sont celles dont le niveau de vie à la retraite est le plus bas.

Décrypter le vieillissement des immigrés sous l'angle de leurs liens familiaux, c'est l'angle d'attaque qu'a choisi Sylvie Emsellem pour appréhender les stratégies familiales des retraités migrants: "La présence ou l'absence de la famille en France, le maintien ou la distance des liens familiaux entre les deux rives de la Méditerranée sont des paramètres fondamentaux pour décrypter la trajectoire des immigrés, notamment à l'heure de la retraite, alors qu'ils résident en France ${ }^{28}$." En s'appuyant sur des entretiens qu'elle a conduits auprès de 85 immigrés, en majorité des Algériens âgés de 55 ans et plus (31 résident seuls en foyer ou en hôtel meublé et 54 vivent en famille), Sylvie Emsellem s'interroge notamment sur le type de relations entretenues avec la famille restée au pays et se demande dans quelle mesure ces relations influencent l'équilibre, la légitimité et les conditions de vie de l'immigré qui vieillit en France. La présence ou l'absence de la famille en France entraînet-elle une forme d'ancrage ou, au contraire, une absence d'attache à la société d'accueil ?

L'une des conclusions majeures de son travail est que les retraités migrants vieillissant en famille et ayant toutes leurs attaches ici préfèrent sans aucun doute rester en France et n'excluent pas, pour une minorité d'entre eux, le fait d'être enterrés en France ${ }^{29}$. Ce qui n'est pas le cas des immigrés ayant laissé femmes et enfants au pays et qui, à défaut de rentrer définitivement, vont trouver dans le vaet-vient entre ici et là-bas une forme d'équilibre à leur condition de retraités. Quant à ceux qui ont rompu tout lien avec leur famille restée au pays, ils demeurent en France dans un isolement total aggravé par des problèmes de santé et des conditions de vie précaires qui les plongent dans une sorte de "mort sociale $\mathrm{e}^{30 "}$.

\section{Bibliographie}

- Aggoun Atmane, "Né ailleurs, vieillissant ici. La poursuite de l'immigration par la retraite", in Annales de la recherche urbaine, $n^{\circ} 100,2006$, pp. 129-135.

- Attias-Donfut Claudine, Daveau Philippe (dir.), L'Enracinement. Enquête sur le vieillissement des immigrés en France, Paris, Armand Colin, 2006.

- Attias-Donfut Claudine, "Les immigrés au temps de la retraite", in Retraite et société, vol. 1, n 44, 2005, p.11-47.

- Attias-Donfut Claudine, Wolf François-Charles, "Transmigration et choix de vie à la retraite", in Retraite et société, vol. 1, n 44, 2005, pp.79-105

- Barou Jacques (dir.), De l'Afrique à la France: d'une génération à l'autre, Paris, Armand Colin, 2011.

- Barou Jacques, "De nouveaux enjeux pour les institutions avec l'arrivée de personnes âgées immigrées", in Soins gérontologie, n 82, 2010, pp. 23-25.

- Blanc-Chaléard Marie-Claude, Histoire de l'immigration, Paris, La Découverte, 2001.

- Bas-Theron Françoise, Michel Maurice, "Rapport sur les immigrés vieillissants", Igas, 2002.

- Bentaleb Nadia, Vieillir dans la dignité. Les immigrés maghrébins vieillissant du centre de Marseille, Paris, Publisud, 2007.

- Bernardot Marc, "Chronique d'une institution: la Sonacotra (1956-1976)", in Sociétés contemporaines, $\mathrm{n}^{\circ} 33-34,1999$, pp. 39-58. 
- Bernardot Marc, "Le vieux, le fou et l'autre. Qui habite encore dans les foyers?”, in Revue européenne des migrations internationales, vol. 1, n 17, 2001, pp. 151-164.

- Berrat Brigitte, "Vieillir dans l'immigration: une vieillesse à part”, in Vie sociale, n³ 3, 2005.

- Bouchaud Olivier, "Le non-retour au pays des migrants âgés : raisons et perspectives", in Soins gérontologie, $n^{\circ} 82,2010$, pp. 35-38.

- Chaïb Yassine, "La mort des immigrés", in Informations sociales, $n^{\circ} 89,2001$, pp.130-143.

- Chaouite Abdelattif, "Accès aux droits et à l'aide sociale des minorités vieillissantes issues de l'immigration", in Vie sociale, n 3, 2005.

- Cheridi Djamel, "Accès aux droits et aux soins dans les foyers de travailleurs migrants", in Écarts d'identité, n 94, 2000, pp. 33-36.

- Glaude Michel, Borrel Catherine, "Les immigrés et leurs descendants sur le marché du travail : un regard statistique", in François Héran (dir.), Immigration, marché du travail, intégration, La Documentation française, Paris, 2002, pp. 105-119.

- Dubus Gilles, Braud Françoise, "Les migrants âgés dans les publications scientifiques francophones", in Revue européenne des migrations internationales, vol. $17, \mathrm{n}^{\circ} 1$, 2001, pp. 189-197.

- Emsellem Sylvie, "Décrypter le vieillissement des immigrés par le prisme de leurs liens familiaux", in L'Année du Maghreb, vol. III, 2007, pp. 601-614.

- Gallou Rémi, "Les immigrés isolés : la spécificité des résidents en foyer", in Retraite et société, $n^{\circ}$ 44, 2005, pp. 108-147.

- Gallou Rémi, "Le vieillissement des immigrés en France : le cas paroxystique des résidents des foyers", in Politix, n 72, 2005, pp. 55-77.

- Hmed Choukri, "Tenir ses hommes. La gestion des étrangers isolés dans les foyers Sonacotra après la guerre d'Algérie", in Politix, n 76, 2006, pp. 11-30.

- Hmed Choukri, "Les immigrés vieillissant en foyer pour travailleurs ou les habitants de nulle part”, in Retraite et société, n 47, 2006, pp. 137-159.
- Jovelin Emmanuel, "Le dilemme des migrants âgés. Entre le désir du retour et la contrainte d'une vie en France", in Pensée plurielle, n 6, 2003, pp. 109-117.

- Kassa Sabrina, Noiriel Gérard, Nos ancêtres les chibanis. Portraits d'Algériens arrivés en France pendant les Trente Glorieuses, Paris, Autrement, 2006.

- Kriegel Blandine, "Le vieillissement des immigrés : un réel enjeu de politique publique", in Retraite et société, n 44, 2005 , pp. 202-206.

- Lestage Françoise, "La mort en migration", in Revue européenne des migrations internationales, vol. 28, n³, 2012, pp. 7-12.

- Math Antoine, "L'accès des vieux migrants aux droits sociaux : un chemin semé d'embûches", 2009, article en ligne sur le site de CATRED : www.catred.org/L-acces-des-vieux-migrantsaux.html

- Meslin Karine, "Des logements à part pour migrants âgés ? Réflexion sur la mise à l'écart résidentielle des migrants âgés isolés", in Espaces, populations, sociétés, 2012, article en ligne http://eps.revues.org/index3974.html

- Pitti Laure, "La main-d'œuvre algérienne dans l'industrie automobile (1945-1962) ou les oubliés de l'histoire", in Hommes \& Migrations, n $1363,2006$.

- Samaoli Omar, "Vieillesse des immigrés. Quelques interrogations d'actualité", in Gérontologie et société, n 139, 2011, pp. 67-75.

- Samaoli Omar, "Mourir dans la différence", in Gérontologie et société, n 91, 1999, pp. 153-155.

- Sayad Abdelmalek, "La vacance comme pathologie de la contradiction d'immigré. Le cas de la retraite et de la préretraite", in Gérontologie, n 60, 1986, pp. 37-55.

- Sayad Abdelmalek, L'Immigration ou les paradoxes de l'altérité, Bruxelles, De Boeck, 1993.

- Sayad Abdelmalek, La Double Absence, Paris, Seuil, 1999.

- Sifaoui Brigitte, "Ces anciens venus d'ailleurs. Quand le soin devient multiculturel”, in Soins gérontologie, n 82, 2010, pp. 17-42. 